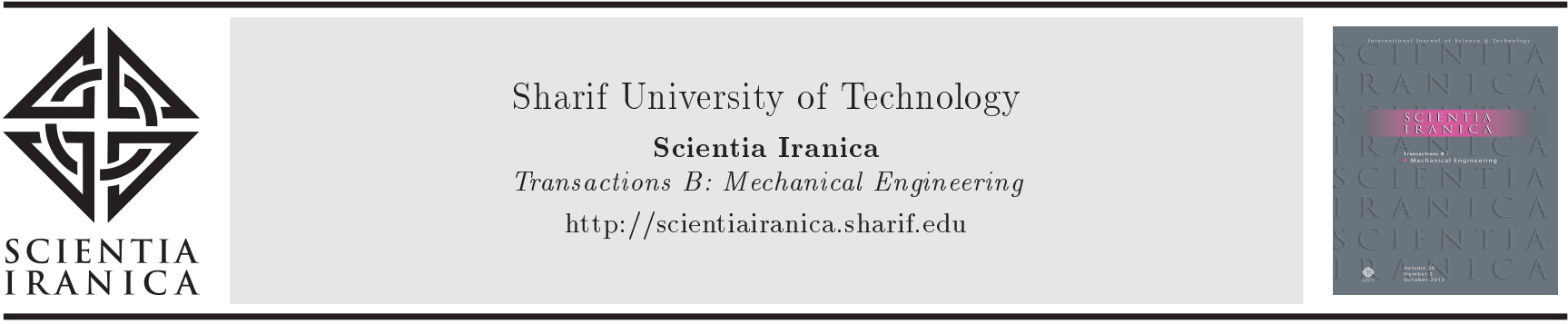

\title{
Numerical investigation of back pressure and free-stream effects on a mixed compression inlet performance
}

\author{
A. Ebrahimi* and M. Zare Chavoshi \\ Department of Aerospace Engineering, Sharif University of Technology, Tehran, P.O. Box. 11155-1639, Iran.
}

Received 9 August 2016; received in revised form 4 January 2017; accepted 25 February 2017

\begin{abstract}
KEYWORDS
Mixed compression

inlet;

Performance

parameters;

Multi-block grid;

Spalart-Allmaras

turbulence model;

Roe's approximated

Riemann solver.
\end{abstract}

\begin{abstract}
Inlet performance has an important role in the operation of air-breathing propulsion systems. In this study, performance of a supersonic axisymmetric mixedcompression inlet in the supercritical operating condition is numerically studied. The effects of free-stream Mach number and engine-face pressure on performance parameters, including mass flow ratio, drag coefficient, total pressure recovery, and flow distortion, are investigated. For this sake, a multi-block density-based finite volume CFD code is developed, and Reynolds-averaged Navier-Stokes equations with Spalart-Allmaras oneequation turbulence model are employed. The code is validated by comparing numerical results against other computational results and experimental data for two test cases of inviscid flow in a two-dimensional mixed-compression inlet and flow in an external compression inlet. Finally, the code is utilized for the investigation of a specific supersonic mixed-compression inlet with the design Mach number of 2.0 and length-to-diameter ratio of 3.4. Results revealed that the increment of free-stream Mach number leads to the decrease in total pressure recovery and drag coefficient, while mass flow ratio and flow distortion increase. The effects of engine-face pressure on performance parameters showed that by increasing the engine-face pressure, mass flow ratio and drag coefficient remain constant, while total pressure recovery increases and flow distortion decreases.
\end{abstract}

(C) 2018 Sharif University of Technology. All rights reserved.

\section{Introduction}

In supersonic flight, engine inlet has a critical role in determining air vehicle performance. An optimum design of an inlet should supply the sufficient air required for the engine with the least amount of total pressure loss and drag generation. Supersonic inlets based on the location of compression shock waves, as shown in Figure 1, are classified into three basic types of internal compression, external compression,

\footnotetext{
*. Corresponding author.

E-mail address: ebrahimi_a@sharif.ir (A. Ebrahimi)
}

doi: $10.24200 /$ sci. 2017.4324 and mixed compression inlets. In internal compression inlets, compression is achieved by a series of oblique shock waves in the internal part of the inlet, following by a weak normal shock downstream of the throat. In external compression inlets, compression occurs by one or more oblique shock waves on the spike, again following by a weak normal shock wave, all of which stand outside the inlet. In this type of inlet, air can also be compressed simply by a normal shock wave. The mixed compression inlet achieves a portion of compression through one or more external oblique shock waves on the spike. The rest of compression occurs in the internal part of the inlet by reflected oblique shocks and the weak normal shock downstream of the throat [1]. 


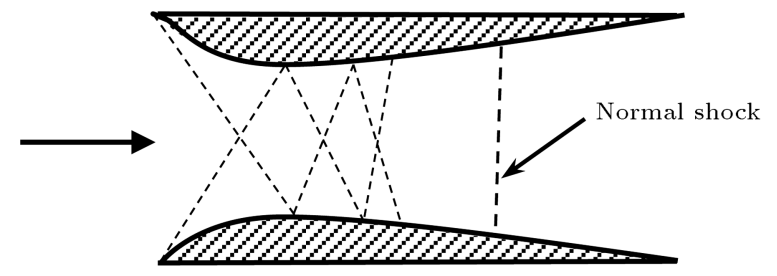

Internal compression

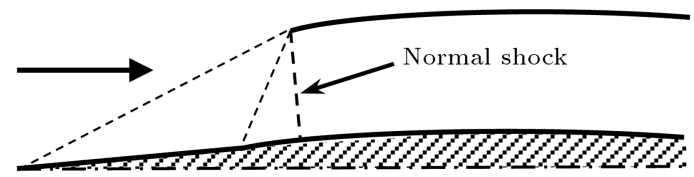

External compression

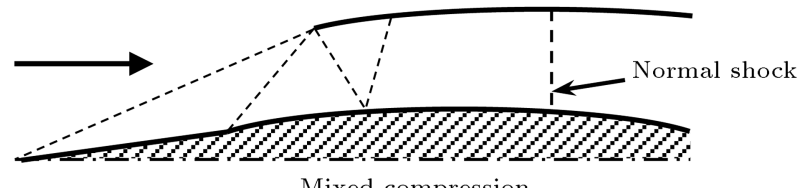

Mixed compression

Figure 1. Types of supersonic inlets.

For Mach numbers above 2.5, external and internal compression inlets become ineffective and fail to meet the minimum requirements of a design. On the other hand, the mixed compression type demonstrates higher efficiency at this range and offers many benefits over the other types. Many investigations were dedicated to mixed compression supersonic inlets in recent years, indicating their popularity amongst designers [2-6]. Chyu et al. [7] considered a three-dimensional unsteady, compressible flow in a mixed compression inlet. They solved Navier-Stokes equations using a combined implicit-explicit (BeamWarming-Steger/MacCormack) method, and BaldwinLomax algebraic method was used for turbulence modeling. Further, they investigated various associated physical phenomena, including shock/boundary layer interaction, subsonic spillage around the cowl lip, and inlet start and unstart. Chan and Liang [8] developed an implicit finite volume code of second-order accuracy in conjunction with the Baldwin-Lomax turbulence model. They simulated a mixed compression inlet near the design point and studied the effects of throat area and bleed on the starting of the inlet. Fujimoto and Niwa [9] numerically and experimentally investigated a supersonic mixed compression inlet of Mach 2.5. They implemented a multi-block finite volume method of second-order accuracy in space and first-order accuracy in time. In addition, Roe's approximate Riemann solver and a $q-\omega$ two-equation turbulence model were employed. They demonstrated that the maximum total pressure recovery of the experimental model and the two-dimensional numerical simulation were different. The reason was argued to be the normal shock boundary-layer interaction considering its sidewall effects. Mizukami and Saunders [10] analyzed a mixed compression inlet using a two-dimensional NavierStokes solver. They performed a parametric study on turbulence models and bleed boundary condition models. Overall, $k-\epsilon$ turbulence model and the bleed boundary condition model based on uniform velocity were recommended because of their good agreement with experimental data. Jain and Mittal [11] solved unsteady Euler equations in a two-dimensional mixed compression supersonic inlet via a stabilized finiteelement method. They analyzed the back pressure and throat area effects on the performance and inlet starting. They reported that, in addition to the throat area, the geometry of the throat plays an important role in starting the process. Akbarzadeh and Kermani [12] numerically simulated inviscid flow in three different types of ramjet inlets. This study was carried out by finite difference method with second-order predictorcorrector scheme of MacCormack and Roe's approximate Riemann solver for inviscid flux calculation. They concluded that the combustion chamber heat rate and the exhaust nozzle throat opening fixed the normal shock wave position in the diffuser. Kwak et al. [13] numerically studied the flow in a mixed compression inlet with bleed regions. Roe's approximate Riemann solver was used for inviscid flux calculation, and two turbulence models of $q-\omega$ and $k-\omega$ SST were used for the evaluation of turbulent flow quantities. Kotteda and Mittal [14] solved unsteady viscous flow in a two-dimensional mixed compression inlet with a stabilized finite-element method. In this study, the effect of bleed on the control of buzz instability and starting/unstarting of the inlet was investigated. Apyan et al. [3] analyzed shock wave boundary-layer interaction in a mixed compression inlet geometry using the OVERFLOW Navier-Stokes equation solver. In addition, they examined the effectiveness of boundary condition to achieve the desired results. Zhao et al. [15] studied the inlet start/unstart phenomena in the acceleration process. They performed unsteady numerical simulation in a two-dimensional mixed compression inlet at Mach number from 1.75 to 2.05 and four different values of acceleration. Kotteda and Mittal [16] examined a turbulent flow in the mixed compression inlet using Spalart-Allmaras turbulence model which highlighted the differences between laminar and turbulent flow simulations. They pointed out the critical back pressure ratio for unstart of the inlet for laminar flow is lower compared to that of turbulent flow.

Literature review indicates that the performance of the mixed compression inlets has not been thoroughly investigated yet. In the previous research by the authors [17], the performance of a mixed compression inlet has been investigated in three free stream Mach numbers. Since inlet performance is under the influence of upstream (free-stream) and also downstream (engine) conditions, a thorough investigation into the 
effects of these conditions on the inlet performance is very important. Mach number is the most important parameter of free-stream flow, and engineface pressure is the main parameter that represents engine performance. Thus, the effects of these two parameters on inlet performance are studied. For this purpose, a structured multi-block density-based finite volume numerical code is developed in order to simulate the axisymmetric flow in a mixed compression supersonic inlet. This code has been validated against two different test cases; finally, performance of an axisymmetric mixed compression inlet with design Mach number of 2.0 was investigated. In this study, the effects of free-stream Mach number and back pressure ratio (static pressure of engine-face to the free-stream static pressure) on performance parameters of the inlet are studied. Supersonic inlet performance parameters include drag coefficient, total pressure recovery, mass flow ratio, and flow distortion.

\section{Performance parameters and numerical methodology}

\subsection{Performance parameters}

The main performance parameters of a supersonic inlet are mass flow ratio, total pressure recovery, drag coefficient, and flow distortion. Mass Flow Ratio (MFR) is the most important characteristic of an inlet as the other performance parameters are dependent on it. By assuming a constant velocity at each section, mass flow ratio is obtained by Eq. (1):

$$
\operatorname{MFR}=\frac{\rho A V}{\rho_{\infty} A_{c} V_{\infty}},
$$

where $\rho, V$, and $A$ are local density, flow velocity, and cross-sectional area inside the inlet, respectively. Also, $\rho_{\infty}, V_{\infty}$, and $A_{c}$ are free-stream density, free-stream velocity, and the cross-section area in the entry face of the inlet, respectively.

Total Pressure Recovery (TPR) of the inlet is another influential characteristic in determining the inlet performance since it has direct effect on the en gine thrust force. Total pressure recovery is defined as the ratio of the total pressure of engine-face $\left(P_{0, f}\right)$ to the total pressure of free-stream $\left(P_{0, \infty}\right)$ flow, shown as follows:

$$
\mathrm{TPR}=\frac{P_{0, f}}{P_{0, \infty}} .
$$

For supersonic inlets, the total drag force is the sum of skin friction drag, pressure drag, and spillage drag. Total drag coefficient is defined as in Eq. (3), in which $D$ is total drag force:

$$
C_{d}=\frac{D}{\frac{1}{2} \rho_{\infty} V_{\infty}^{2} A_{c}} .
$$

Inlets are usually exposed to an adverse pressure gradient, which results in flow separation. Flow separation causes total pressure loss and nonuniformity of total pressure distribution in each section. This phenomenon is called Flow Distortion (FD), and it is quantified in Eq. (4) as follows:

$$
\mathrm{FD}=\frac{P_{0, \max }-P_{0, \min }}{P_{0, a v g}} .
$$

Since the uniformity of engine inflow is of the greatest importance, usually, the flow distortion of the engine face is evaluated.

\subsection{Governing equations}

In this investigation, two-dimensional (planar and axisymmetric) compressible Navier-Stokes equations are employed. The conservation form of these equations with no body force and heat addition is stated in Eq. (5):

$$
\frac{\partial \vec{U}}{\partial t}+\frac{\partial \vec{E}}{\partial x}+\frac{\partial \vec{F}}{\partial y}+\frac{\partial \overrightarrow{E_{v}}}{\partial x}+\frac{\partial \overrightarrow{F_{v}}}{\partial y}+\alpha \vec{V}+\alpha \overrightarrow{V_{v}}=0
$$

where $\vec{U}$ is conservative variables vector, $\vec{E}$ and $\vec{F}$ are inviscid flux vectors, $\overrightarrow{E_{v}}$ and $\overrightarrow{F_{v}}$ are viscous flux vectors, and $\vec{V}$ and $\vec{V}_{v}$ are vectors of axisymmetric flow source terms that are represented as in Eq. (6). Moreover, $\alpha$ determines the two dimensionality $(\alpha=0)$ or axisymmetry $(\alpha=1)$ of equations:

$$
\begin{aligned}
& \vec{U}=\left[\begin{array}{c}
\rho \\
\rho u \\
\rho v \\
\rho E
\end{array}\right], \vec{E}=\left[\begin{array}{c}
\rho u \\
P+\rho u^{2} \\
\rho u v \\
(P+\rho E) u
\end{array}\right], \vec{F}=\left[\begin{array}{c}
\rho v \\
\rho u v \\
P+\rho v^{2} \\
(P+\rho E) v
\end{array}\right], \\
& \vec{V}=\frac{1}{y}\left[\begin{array}{c}
\rho v \\
\rho u v \\
\rho v^{2} \\
\rho v H
\end{array}\right], \overrightarrow{E_{v}}=\left[\begin{array}{c}
0 \\
-\tau_{x x} \\
-\tau_{x y} \\
-u \tau_{x x}-v \tau_{x y}+q_{x}
\end{array}\right], \\
& \overrightarrow{F_{v}}=\left[\begin{array}{c} 
\\
-\tau_{x y} \\
-\tau_{y y} \\
-u \tau_{x y}-v \tau_{y y}+q_{y}
\end{array}\right], \\
& \overrightarrow{V_{v}}=\frac{1}{y}\left[\begin{array}{c}
0 \\
-\tau_{y y}+\tau_{\theta \theta} \\
-u \tau_{x y}-v \tau_{y y}+q_{y}
\end{array}\right] .
\end{aligned}
$$

In the above equations, $\rho, u, v, E$, and $H$ represent density, axial velocity (or $x$-velocity) component, radial velocity (or $y$-velocity) component, total energy, and total enthalpy, respectively. Also, $\tau_{i j}$ and $q_{i}$ are stress tensor and heat transfer vector. Details of governing equation terms are completely described in [18]. 


\subsection{Turbulence modeling}

Averaging the Navier-Stokes equations produces the so-called Reynolds stress tensor, which leads to the turbulence closure problem. Boussinesq's hypothesis treats Reynolds stress as the molecular stress and, finally, computes the turbulence eddy viscosity by different turbulence models [19]. Among different turbulence models, Spalart-Allmaras model is a oneequation model that has been developed specifically for aerospace applications involving wall-bounded flows, and it has shown approving results for boundary layers with adverse pressure gradients [20]. Although there are turbulence models (e.g., LES and DES) that are more accurate than the Spalart-Allmaras model, these models are computationally expensive. Since the detailed information of the flow field is not required in the performance study, a far simplified model needs to be devised in order to have both acceptable accuracy and fast simulation times. Besides, many of performed numerical studies on supersonic inlet have implemented this model [16,21-23]. Conclusively, the Spalart-Allmaras model was chosen for this investigation.

Working variable of Spalart-Allmaras model indicates turbulent kinematic viscosity, everywhere except the regions near walls where the influence of viscosity is dominant. Transport equation of this working variable is written as in Eq. (7) [24]:

$$
\frac{\partial}{\partial t}(\bar{\rho} \hat{v})+\frac{\partial}{\partial x_{j}}\left(\bar{\rho} \tilde{u_{i}} \hat{v}\right)=S_{\text {Diff. }}+S_{\text {Prod. }}-S_{\text {Dist. }},
$$

where $S_{\text {Diff. is diffusion term, }} S_{\text {Prod. }}$ is production term, and $S_{\text {Dist. }}$ represents destruction term, stated as in Eqs. (8) to (10):

$$
\begin{aligned}
& S_{\text {Prod. }}=c_{b 1} \bar{\rho} \hat{S} \hat{v} \\
& S_{\text {Dist. }}=c_{w 1} \bar{\rho} f_{w}\left(\frac{\hat{v}}{d}\right)^{2}, \\
& S_{\text {Diff. }}=\frac{\partial}{\partial x_{j}}\left(\mu_{\text {eff }} \frac{\partial \hat{v}}{\partial x_{j}}\right)+\frac{\bar{\rho} c_{b 2}}{\sigma} \frac{\partial \hat{v}}{\partial x_{j}} \frac{\partial \hat{v}}{\partial x_{j}} .
\end{aligned}
$$

Turbulent viscosity, $\mu_{t}$, is obtained from Eq. (11), while functions in Eqs. (12)-(20) are also used:

$$
\begin{aligned}
& \mu_{t}=\bar{\rho} \hat{v} f_{v 1}, \\
& f_{v 1}=\frac{\chi^{3}}{\chi^{3}+c_{v 1}^{3}}, \\
& \chi=\frac{\hat{v}}{v}, \\
& f_{v 2}=1-\frac{\chi}{1+\chi f_{v 1}},
\end{aligned}
$$

$$
\begin{aligned}
& f_{w}=g\left(\frac{1+c_{w 3}^{6}}{g^{6}+c_{w 3}^{6}}\right), \\
& g=r+c_{w 2}\left(r^{6}-r\right), \\
& r=\frac{\hat{v}}{\hat{S} \kappa^{2} d^{2}}, \\
& S=\sqrt{2 \Omega_{i j} \Omega_{i j}}, \\
& \bar{S}=\frac{\hat{v}}{\kappa^{2} d^{2}} f_{v 2}, \\
& \left\{\begin{array}{l}
S+\bar{S} \\
s+\frac{S\left(c_{v 2}^{2} S+c_{v 3} \bar{S}\right)}{\left(c_{v 3}-2 c_{v 2}\right) S-\bar{S}} \quad: \bar{S} \geq-c_{v 2} \\
\end{array}\right.
\end{aligned}
$$

The constants are given as in Eq. (21) taken from [24]:

$$
\begin{aligned}
& \kappa=0.4187, \quad \sigma=\frac{2}{3}, \quad c_{b 1}=0.1355, \quad c_{b 2}=0.622, \\
& c_{w 1}=\frac{c_{b 1}}{\kappa^{2}}+\frac{\left(1+c_{b 2}\right)}{\sigma}, \quad c_{w 2}=0.3, \\
& c_{w 3}=2.0, \quad c_{v 1}=7.1, \quad c_{v 2}=0.7, \quad c_{v 3}=0.9 .
\end{aligned}
$$

\subsection{Numerical method}

A cell-centered density-based finite volume method in a structured multi-block grid is used in which data transformation between blocks is performed by ghost cells with second-order accuracy, and inter-block faces have the same grid points. Explicit six-stage Runge-Kutta method was employed for time discretization. Also, the local time stepping method is used for convergence acceleration to steady-state solution. In this method, the solution of each cell is advanced in time with the local time step obtained by the local stability limit of that cell. Roe's approximated Riemann solver [25] is used for calculating inviscid fluxes, while the central difference method is employed to compute the viscous fluxes. Further, van Leer's Monotonic Upstreamcentered Scheme for Conservation Laws (MUSCL) extrapolation [26] with the van Albada's limiter is used to obtain second-order accuracy while maintaining Total Variation Diminishing (TVD) property.

\section{Validation of numerical code}

\subsection{Inviscid flow around two-dimensional mixed compression supersonic inlet}

This test case was used to evaluate the numerical code in the calculation of inviscid fluxes and data transfer between the computational domain blocks. The geometry of this test case is a mixed compression inlet with the design Mach number of 3.0 and with two ramps. The first ramp has an angle of 7 degrees and a length of 28 inches, and the second one has an 


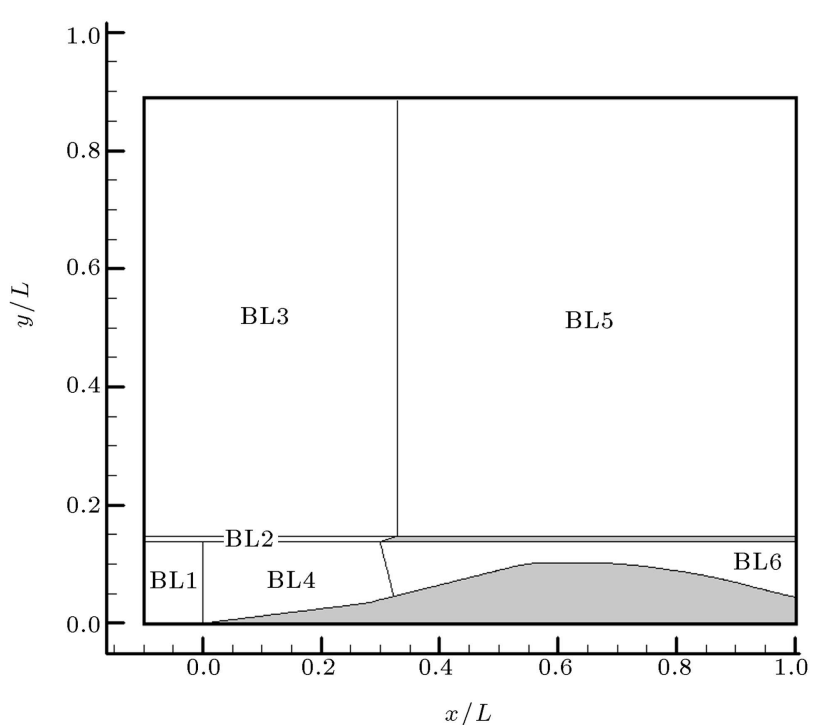

(a)

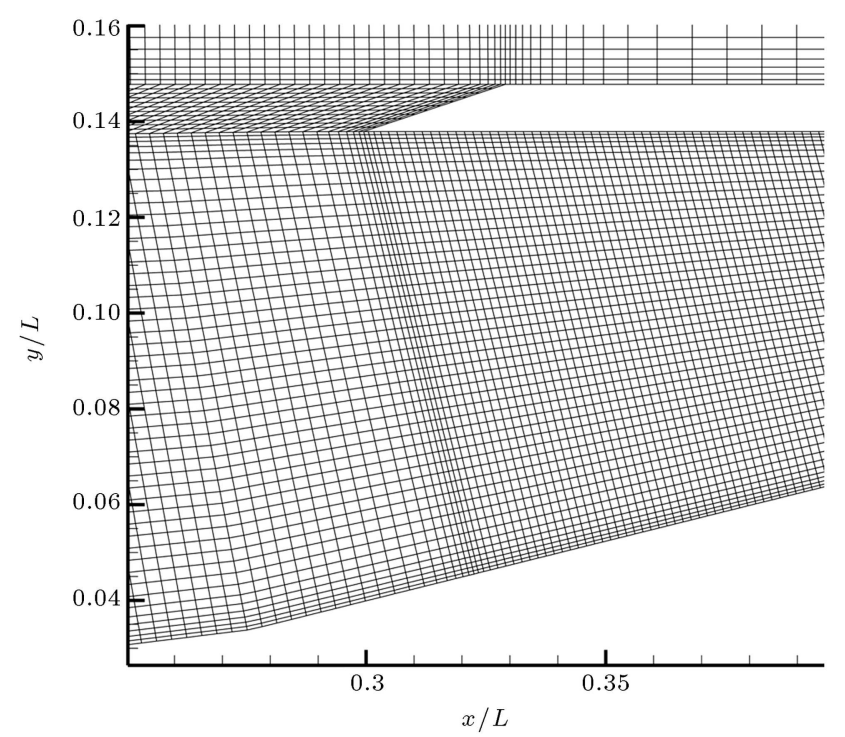

(b)

Figure 2. (a) Configuration of inlet. (b) Computational grid.

inclination of 14 degrees with a length of 24.1 inches. Total length is 101.15 inches that is considered as the characteristic length for normalizing other lengths of the inlet. This inlet geometry was experimentally studied previously by Anderson and Wong [27], and then many researchers performed numerical studies on it. At first, Jain and Mittal [11] implemented the finiteelement method to solve inviscid flow in the modified geometry, and then Kwak et al. [13] used finite volume method for this purpose. The schematic of this inlet in conjunction with the computational domain blocks is sketched in Figure 2(a). The domain has been divided into 6 blocks that have 34,882 computational cells altogether. A magnified view of the computational grid around the ramp and cowl lip is illustrated in

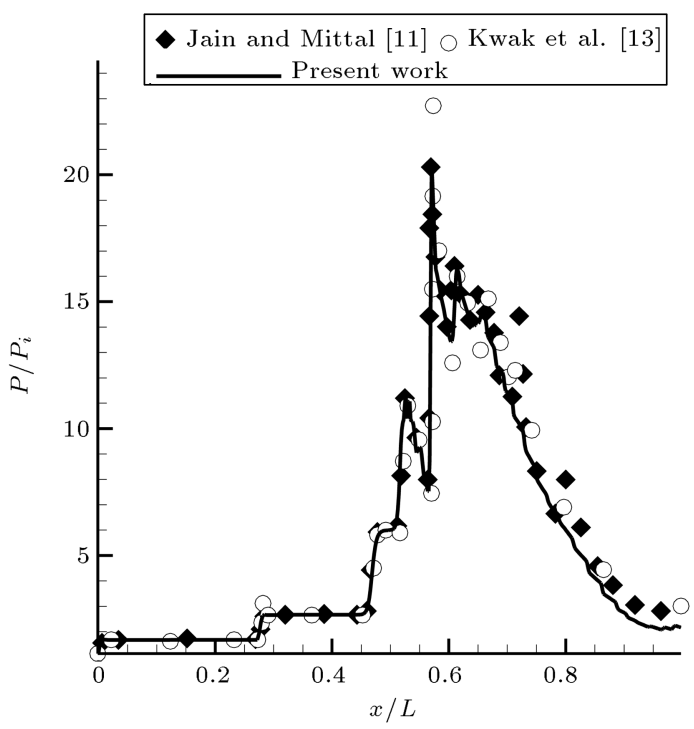

Figure 3. Pressure distribution on the spike.

Figure 2(b). Figure 3 shows the normalized pressure distribution over the spike resulting from this work along with numerical results of Jain and Mittal [11] and Kwak et al. [13]; evidently, the results are in good agreement with each other.

\subsection{Turbulent flow around an axisymmetric external compression inlet}

In this section, the capability of this code in solving the axisymmetric flow of an external compression supersonic inlet is evaluated. The flow of an external compression inlet is similar to that of a mixed compression inlet, and the performance of the numerical code is evaluated in the presence of phenomena, such as the shock wave and its interactions with turbulent boundary layer and shock-induced separation. Further, due to the complicated shape of the inlet, the multiblock grid is employed in which the ability of the code in data transferring between the blocks is examined. This inlet has a design Mach number of 2.0 with a length-to-diameter ratio of 4.8 that was designed by Soltani and Farahani [28] to investigate the buzz phenomenon of supersonic inlets.

In the present work, the flow field around the inlet is divided into six blocks with a total of 35,747 computational cells. The numerical solution was performed for a free-stream Mach number of 2.0, static pressure of 10,799.5 Pa., static temperature of 167.24 Kelvin, $\hat{\nu}_{\infty} / \nu_{\infty}=3.0$, and engine-face static pressure of $31,966.5 \mathrm{~Pa}$. The results are compared with the experiments presented by Soltani et al. [29].

Static pressure distribution over the spike and total pressure profile at the throat $(x / d=0.85)$ are plotted in Figures 4 and 5, respectively. According to the plots, numerical results show great consistency with experimental results. 


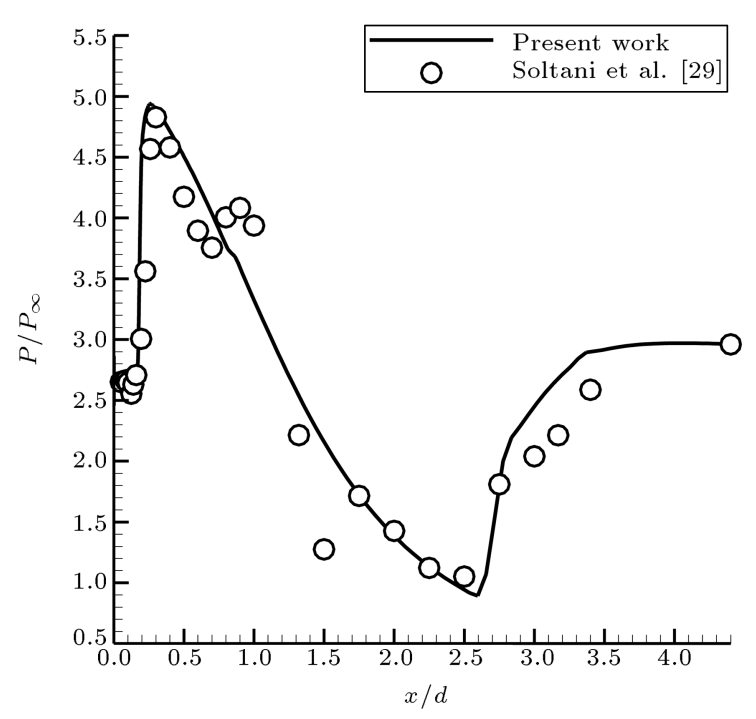

Figure 4. Distribution of static pressure on spike.

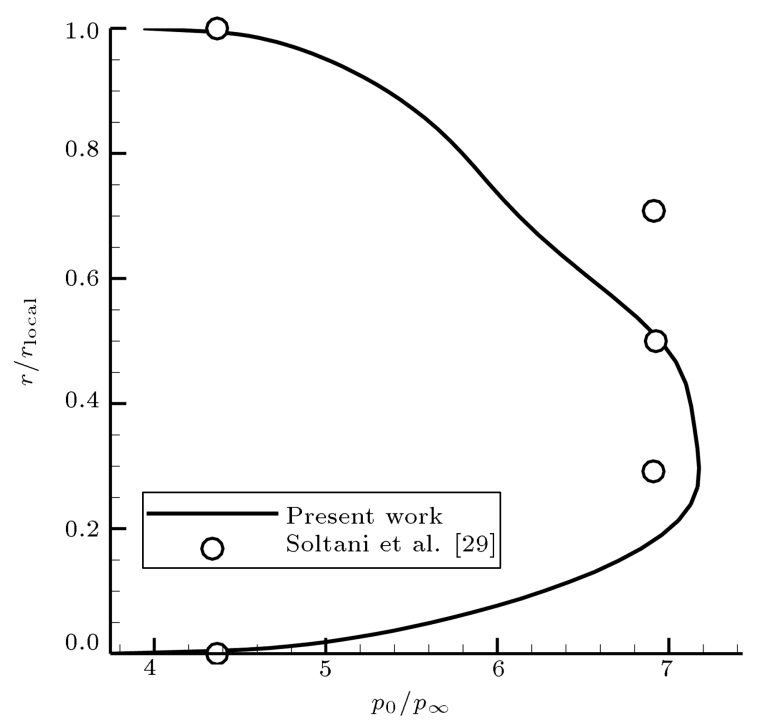

Figure 5. Stagnation pressure profile at throat, $x / d=0.82$

\section{Results and discussion}

\subsection{The mixed compression supersonic inlet}

The mixed compression inlet of this study is an axisymmetric supersonic inlet that is designed for the Mach number of 2.0 with a length-to-diameter ratio of 3.4. This inlet has been designed and experimentally studied for the first time by Soltani et al. [30]. In order to study the flow field of this inlet, a hyperbolic computational domain is considered ahead of the inlet. The height of domain is selected large enough to prohibit shock waves from hitting the computational domain boundary. To generate a high-quality grid, the computational domain is divided into six blocks, as shown in Figure 6.

Grid convergence study was performed in the previous work [17]; finally, the mesh with total cells'

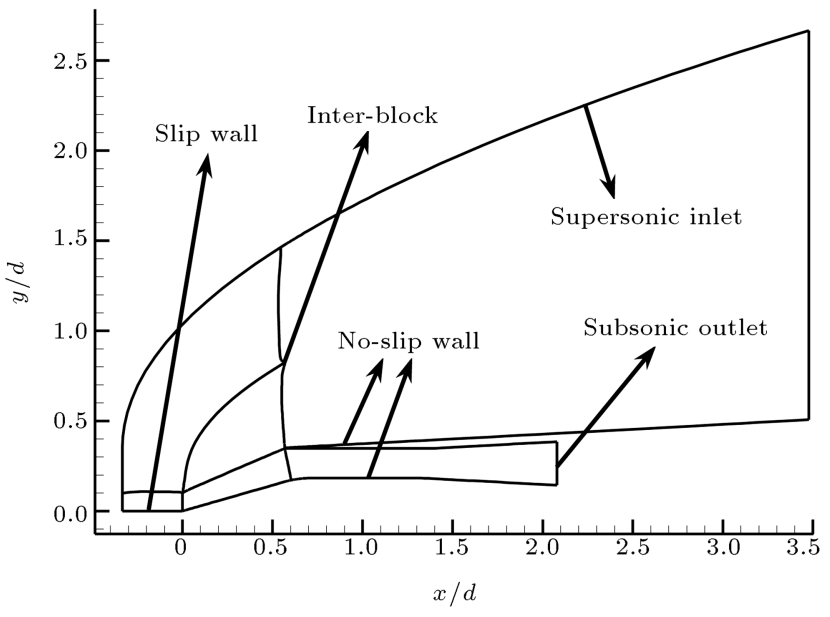

Figure 6. Computational blocks and boundary conditions.

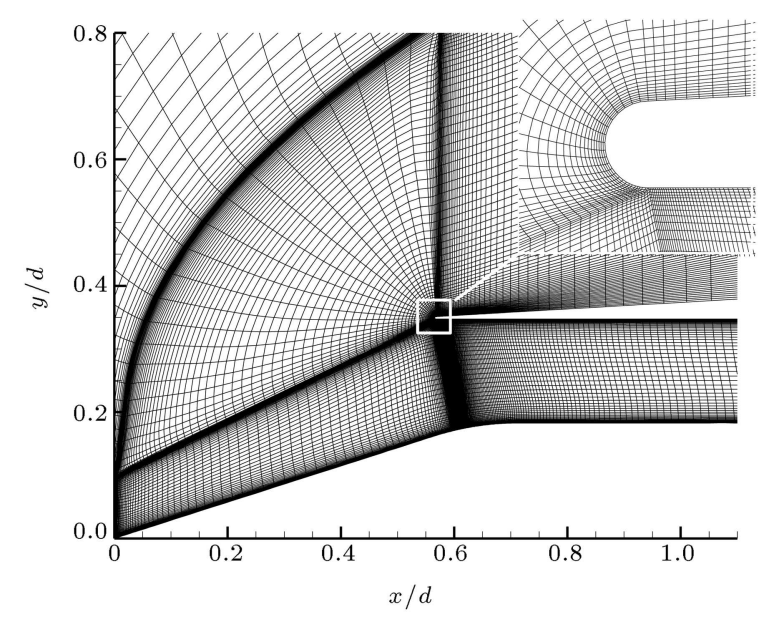

Figure 7. Magnified view of optimum computational grid.

number of 35,747 was chosen as the optimum one for the computational solution. A view of this mesh around the spike and cowl lip is illustrated in Figure 7.

In the following, the flow field in the inlet has been solved for the Mach number of 2.0, Reynolds number of $1.15 \times 10^{6}, \hat{\nu}_{\infty} / \nu_{\infty}=3.0$, and back pressure ratio of 4.75 . To gain a better appreciation of flow characteristics of the inlet, pressure coefficient contours are shown in Figure 8. According to this plot, a conical shock wave is formed in front of the spike and Mach number decreases across it, though flow remains supersonic. Then, considering the upstream of the throat at the edge of spike curvature, establishment of Prandtl-Mayer expansion waves impinges on the shock wave formed from cowl lip and weakens it. The continuous intersection of oblique shock and expansion waves and their reflection from walls is afflicted by flow Mach number; eventually, it ends with a weak normal shock downstream of the throat.

\subsection{Effects of free-stream Mach number}

In this section, the effects of free-stream Mach number 


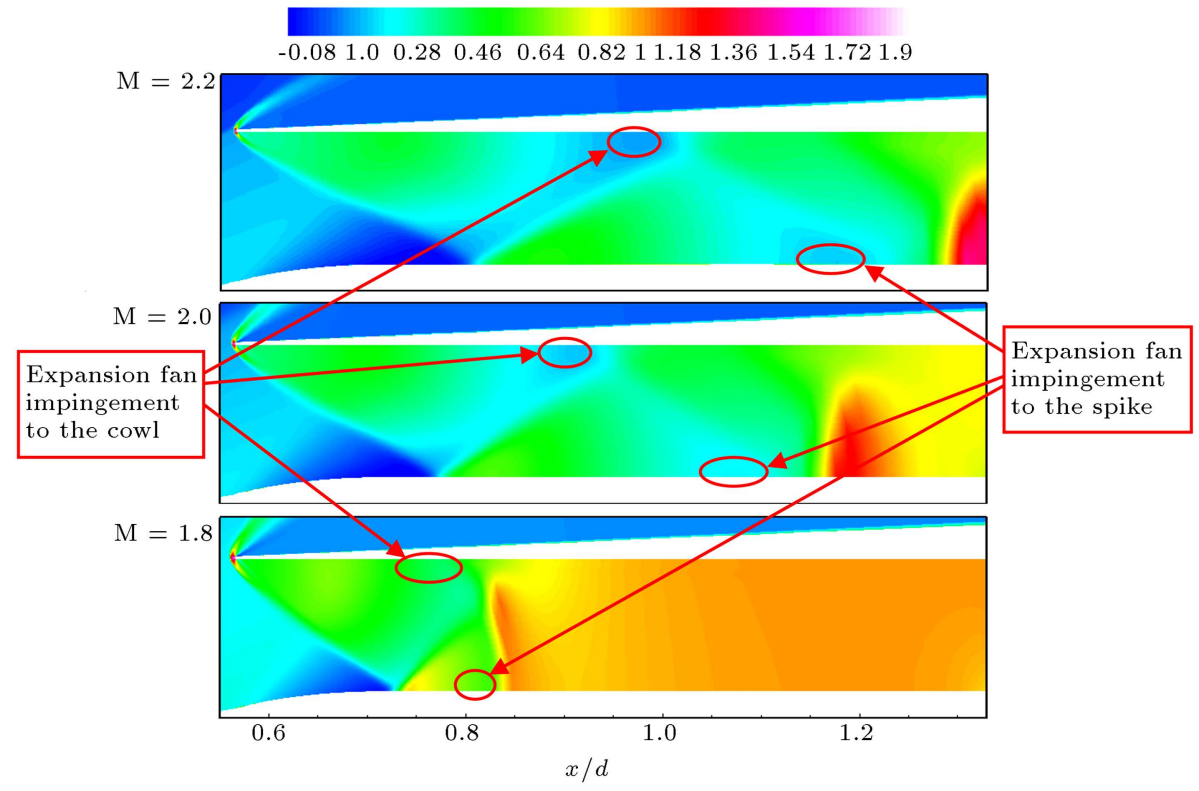

Figure 8. Pressure coefficient contours for Mach numbers of 1.8, 2.0, and 2.2.

on the performance of this special inlet are studied at constant back pressure ratio in the supercritical operating condition. This investigation has been performed on various Mach numbers from 1.8 to 2.2, at the Reynolds number of $1.15 \times 10^{6}, \hat{\nu}_{\infty} / \nu_{\infty}=3.0$, and back pressure ratio of 4.75 . Pressure coefficient contours for Mach numbers of 1.8, 2.0, and 2.2 are shown in Figure 8, and Pressure coefficient distribution over spike surface is demonstrated in Figure 9 . It is seen that, for all Mach numbers, the normal shock wave is established in diffuser, while, for the Mach number of 1.8 , it is closer to the throat. By increasing the free-stream Mach number, this normal shock wave moves downstream and, consequently, as the cross-

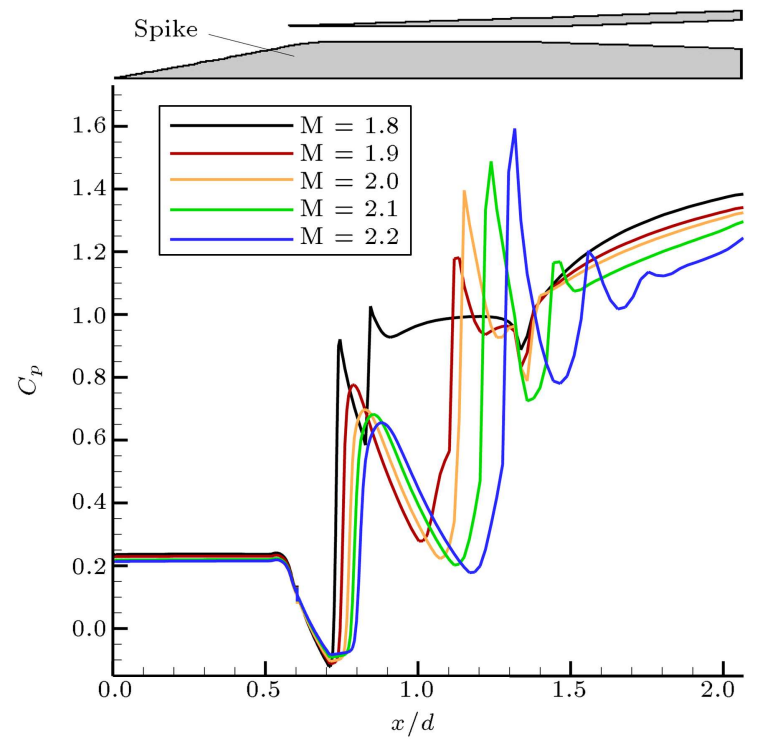

Figure 9. Pressure coefficient distribution over spike at different Mach numbers. section area gets bigger (higher local Mach number), shock wave becomes stronger. Moreover, as the local Mach number at upstream increases, the expansion waves emanating from spike (at $x / d=0.65$ ) become stronger. Therefore, the reflection of these waves that hits the spike surface again move further downstream. As presented in Figure 8, at Mach numbers of 1.8, 2.0, and 2.2 , these waves impinge on the spike at $x / d=0.8$, 1.1 , and 1.2 , respectively.

Variations of mass flow ratio and drag coefficient versus those of Mach number are illustrated in Figure 10. It is shown that by increasing the freestream Mach number, mass flow ratio increases and drag coefficient decreases. This is because as freestream Mach number increases, the angle of established oblique shock wave in front of the spike decreases. Consequently, the oblique shock becomes closer to the cowl lip and mass flow spillage decreases. By the reduction of spillage flow, a higher mass flow enters the inlet, and conclusively spillage drag and total drag coefficient decreases. As it is obvious from Figure 10, when Mach number is 2.2 , the mass flow ratio is nearly equal to 1 , which indicates the situation that oblique shock wave is placed exactly on the cowl lip and no flow spillage occurs.

Further, by increasing the free-stream Mach number from 1.8 to 2.2 , mass flow ratio is increased by $8 \%$, while drag coefficient has a decrement of about $30 \%$. This fact indicates the significant effect of mass flow ratio on inlet drag coefficient.

Variations of two performance parameters, i.e. total pressure recovery and flow distortion, are sketched against Mach number in Figure 11. As seen, by increasing Mach number, total pressure recovery decreases and flow distortion increases. With the movement 


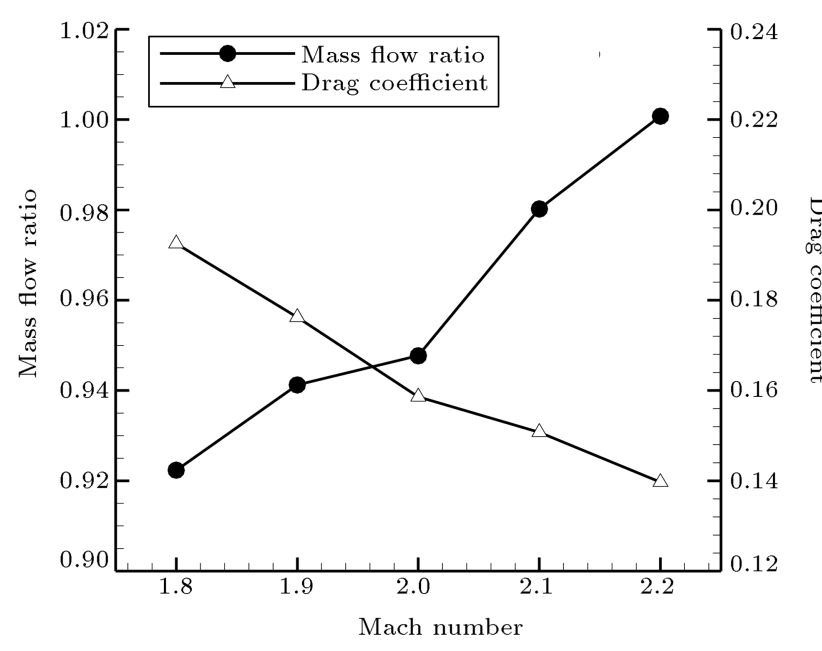

Figure 10. Variations of mass flow ratio and drag coefficient versus those of Mach number.

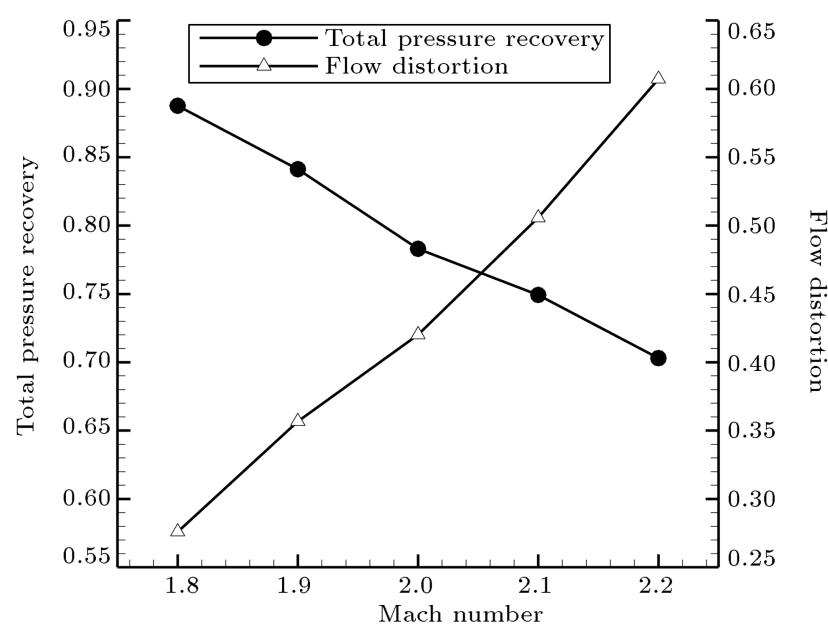

Figure 11. Variations in total pressure recovery and flow distortion against those in Mach number.

of the normal shock wave towards downstream, its strength intensifies and total pressure loss increases. Moreover, because of the stronger normal shock, the boundary layer is exposed to a higher adverse pressure gradient, and consequently, a larger separation region is formed. Therefore, by increasing the free-stream Mach number, these two factors lead to a reduction in total pressure and total pressure recovery. Since the strength of normal shock wave and flow separation are highly dependent upon the position of normal shock wave and the shape of the diffuser, the variations of total pressure recovery with Mach number can differ significantly for various inlets. As seen in Figure 11, pressure recovery has decreased 20\% when the free-stream Mach number varied from 1.8 to 2.2. In addition, Figure 11 reveals that flow distortion at engine-face has been severely changed by Mach number so that, with Mach number increment from 1.8 to 2.2 , flow distortion has increased up to $100 \%$. Enlargement of separation region results in bigger loss of total pressure in the affected zones in comparison with other regions. That zone deteriorates the flow uniformity and increases flow distortion with Mach number increment. It is worth emphasizing that variations of flow distortion with Mach number are a function of diffuser shape and can be quite different for various inlets. It is noted that these findings for mixed compression inlet in the supercritical operating condition are consistent with the study done by Soltani et al. [31] for external compression inlet under the similar operating condition.

\subsection{Engine-face pressure effects}

In the real operational condition, mass flow rate of the inlet is restricted by engine performance; this restriction leads to a pressure variation at downstream of the inlet that affects its performance. Therefore, in this section, the effect of back pressure in the supercritical operating condition is investigated in order to study the effect of engine operation on the inlet performance. This is conducted on Reynolds number of $1.15 \times 10^{6}, \hat{\nu}_{\infty} / \nu_{\infty}=3.0$, Mach number of 2.0 , and five back pressure ratios of $3.65,4.1,4.75,5.1$, and 5.5.

Pressure coefficient distribution over the spike is plotted for various back pressure ratios in Figure 12. In addition, variations of performance parameters against back pressure ratio are sketched in Figures 13 and 14 . According to Figure 12, by increasing the back pressure ratio, the normal shock wave moves upstream; however, due to the supersonic speed of upstream flow, the condition upstream of the normal shock remains unchanged. Plots of performance parameters also show a negligible difference in the mass flow passing through the inlet. Further, drag coefficient remains nearly invariant since the mass flow passing through the inlet

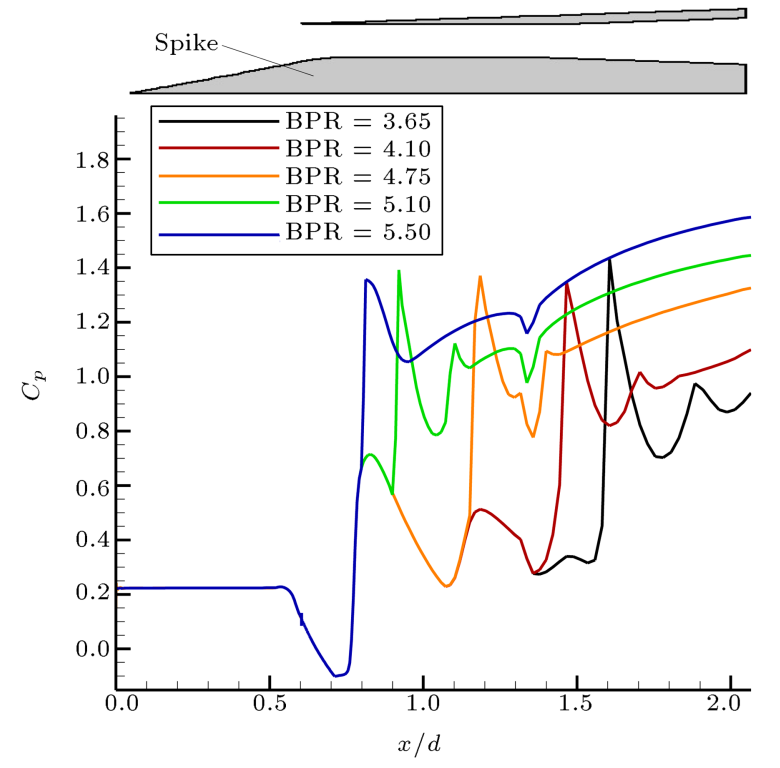

Figure 12. Pressure coefficient distribution over spike at various back pressure ratios. 


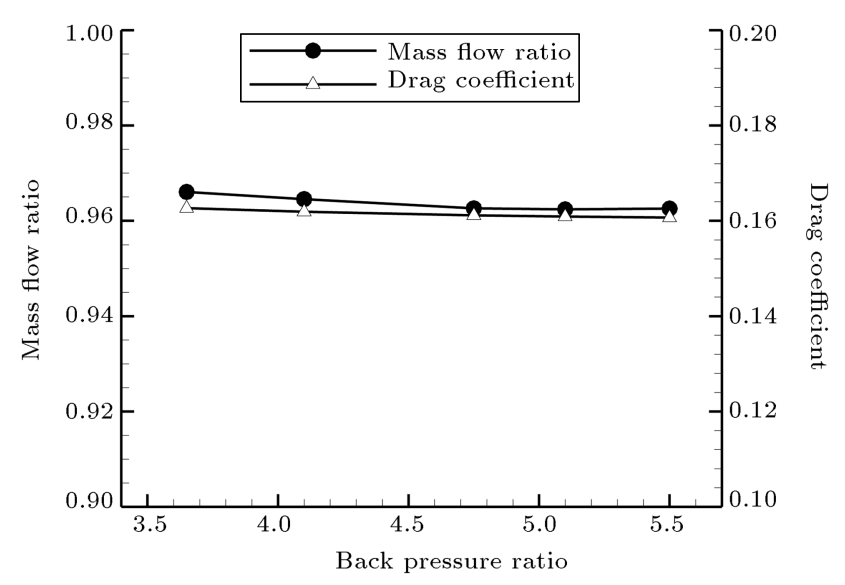

Figure 13. Variations in mass flow ratio and drag coefficient versus those in back pressure ratio.

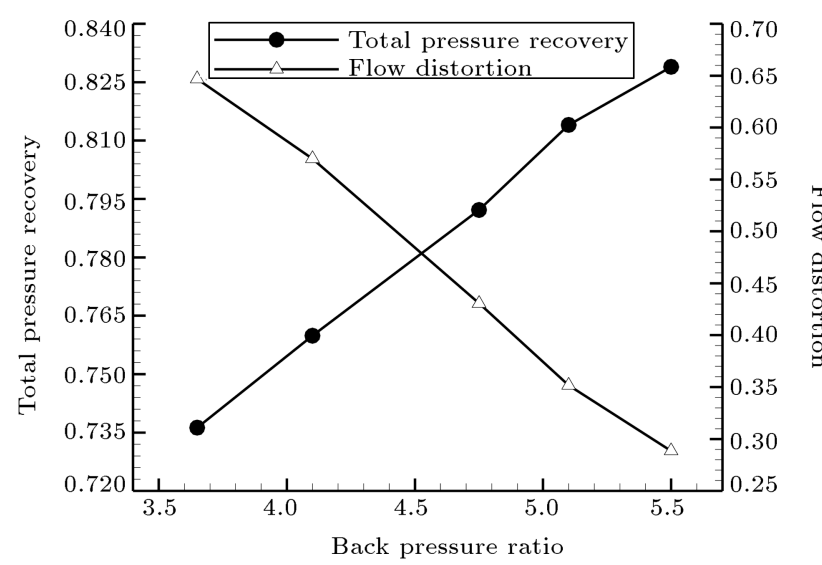

Figure 14. Variations of total pressure recovery and flow distortion against those of back pressure ratio.

is constant, that is consistent with the literature [31] for the supercritical operating condition.

With increasing back pressure ratio, the normal shock wave moves upstream where cross-section area is smaller. Consequently, local Mach number decreases and the normal shock wave becomes weaker. Therefore, both stagnation pressure loss and flow separation decrease. As a result, with increasing back pressure ratio, pressure recovery and flow distortion increase and decrease, respectively.

\section{Conclusions}

In this study, a numerical code was developed using the density-based finite volume method. A structured multi-block grid and an explicit time discretization of Reynolds-Averaged Navier-Stokes (RANS) equations were used. Furthermore, Roe's approximated Riemann solver was utilized for computing inviscid flux vectors. In addition, the Monotone Upstream centered Schemes for Conservation Laws (MUSCL) extrapolation with van Albada limiter were used to obtain second-order accuracy. In addition, Spalart-Allmaras one-equation turbulence model was used to close the governing equations. The code was validated by two different test cases. Then, the axisymmetric flow around a mixed compression supersonic inlet was studied, and the effects of free stream Mach number and back pressure ratio on the performance parameters, including mass flow ratio, drag coefficient, total pressure recovery, and flow distortion, were investigated in detail. The results revealed that by increasing the free-stream Mach number, the angle of oblique shock wave established in front of the spike reduces, mass flow ratio increases, and consequently, spillage drag and total drag coefficient decrease. Moreover, increasing the free-stream Mach number causes normal shock wave to move downstream, which results in an increment of flow distortion and a reduction in total pressure recovery. The effects of back pressure ratio on performance parameters showed that by increasing the back pressure ratio, mass flow ratio and drag coefficient remain constant as the flow structure upstream of the throat is nearly unchanged in the supercritical operating condition. However, normal shock wave moves upstream and its strength decreases. Therefore, total pressure recovery increases and flow distortion decreases.

\section{References}

1. Mattingly, J.D., Heiser, W.H., and Pratt, D.T., Aircraft Engine Design, New York: American Institute of Aeronautics and Astronautics (2002).

2. Xie, L. and Guo, R. "Investigation of a twodimensional mixed-compression hypersonic inlet", Hangkong Xuebao/Acta Aeronautica et Astronautica Sinica, 30(12), pp. 2288-2294 (2009).

3. Apyan, A.C., Orkwis, P.D., Turner, M.G., Duncan, S., Benek, J., and Tinnaple, J. "Mixed compression inlet simulations with aspiration", in 50th AIAA Aerospace Sciences Meeting Including the New Horizons Forum and Aerospace Exposition, Nashville, TN (2012).

4. Vaynshtein, A. and Arieli, R. "Start/Un-start process for a sudden opening of a mixed compression inlet system of a ramjet engine", in 54th Israel Annual Conference on Aerospace Sciences, IACAS 2014, TelAviv and Haifa, pp. 899-920 (2014).

5. Yongzhao, L., Qiushi, L., and Shaobin, L. "Modeling the effect of stability bleed on back-pressure in mixedcompression supersonic inlets", Journal of Fluids Engineering, Transactions of the ASME, 137(12), 121101 (2015).

6. Qiushi, L., Yongzhao, L., and Shaobin, L. "A quasi one-dimensional bleed flow rate model for terminal 
normal shock stability in mixed compression supersonic inlet", Proceedings of the Institution of $\mathrm{Me}$ chanical Engineers, Part C: Journal of Mechanical Engineering Science, 228(14), pp. 2569-2583 (2014).

7. Chyu, W.J., Kawamura, T., and Bencze, D.P. "Calculation of external-internal flow fields for mixedcompression inlets", Computer Methods in Applied Mechanics and Engineering, 64(1-3), pp. 21-37 (1987).

8. Chan, J.-J. and Liang, S.-M. "Numerical investigation of supersonic mixed-compression inlet using an implicit upwind scheme", Journal of Propulsion and Power, 8(1), pp. 158-167 (1992).

9. Akira, F. and Nobuo, N. "Experimental and numerical investigation of Mach 2.5 supersonic mixed compression inlet", in 31st Aerospace Sciences Meeting, Reno, NV, U.S.A. (1993).

10. Mizukami, M. and Saunders, J. "Parametrics on 2D Navier-Stokes analysis of a Mach 2.68 bifurcated rectangular mixed-compression inlet", 31st Joint Propulsion Conference and Exhibit, American Institute of Aeronautics and Astronautics (1995).

11. Jain, M.K. and Mittal, S. "Euler flow in a supersonic mixed-compression inlet", International Journal for Numerical Methods in Fluids, 50(12), pp. 1405-1423 (2006).

12. Akbarzadeh, M. and Kermani, M. "Numerical simulations of inviscid airflows in ramjet inlets", Transactions of the Canadian Society for Mechanical Engineering, 33(2), pp. 271-296 (2009).

13. Kwak, E. Lee, H., and Lee, S. "Numerical simulation of flows around axisymmetric inlet with bleed regions", Journal of Mechanical Science and Technology, 24(12), pp. 2487-2495 (2011).

14. Kotteda, V.M.K. and Mittal, S. "Viscous flow in a mixed compression intake", International Journal for Numerical Methods in Fluids, 67(11), pp. 1393-1417 (2011).

15. Zhao, H., Xie, L.R., Guo, R.W., Tneg, Y.L., and Zhang, J. "Study of start/unstart phenomenon of supersonic inlet in acceleration/deceleration process", Hangkong Dongli Xuebao/Journal of Aerospace Power, 30(8), pp. 1841-1852 (2015).

16. Kotteda, V.M.K. and Mittal, S. "Computation of turbulent flow in a mixed compression intake", International Journal of Advances in Engineering Sciences and Applied Mathematics, 6(3), pp. 126-141 (2015).

17. Ebrahimi, A. and Zare Chavoshi, M. "Effect of free stream Mach number on a mixed compression inlet performance", Modares Mechanical Engineering, 16(7), pp. 275-284, (2016). (in Persian)

18. Suzen, Y.B. "Numerical computation of compressible, turbulent high-Speed flows", 9900518 Thesis, Wichita State University, Ann Arbor (1998).

19. Blazek, J. "Turbulence modelling", in: Computational Fluid Dynamics: Principles and Applications, Chapter 7, 2nd Edn., pp. 227-270, Oxford: Elsevier Science (2005).

20. Spalart, P.R. and Allmaras, S.R. "A one-equation turbulence model for aerodynamic flows", 30th Aerospace Sciences Meeting and Exhibit, Reno, NV, U.S.A. (1992).

21. Slater, J.W. "Verification assessment of flow boundary conditions for CFD analysis of supersonic inlet flows", NASA TM 2012-211790, National Aeronautics and Space Administration, Glenn Research Center (2002).

22. Kim, H., Kumano, T., Liou, M.S., Povinelli, L.A., and Conners, T.R. "Flow simulation of supersonic inlet with bypass annular duct", Journal of Propulsion and Power, 27(1), pp. 29-39 (2011).

23. Kwak, E. and Lee, S. "Numerical study of the effect of exit configurations on supersonic inlet buzz", 31st AIAA Applied Aerodynamics Conference, San Diego, CA (2013).

24. Allmaras, S.R. and Johnson, F.T. "Modifications and clarifications for the implementation of the SpalartAllmaras turbulence model", In Seventh International Conference on Computational Fluid Dynamics (ICCFD7), Big Island, Havaii, pp. 1-11 (2012).

25. Roe, P.L. "Approximate Riemann solvers, parameter vectors, and difference schemes", Journal of Computational Physics, 43(2), pp. 357-372 (1981).

26. Van Leer, B. "Towards the ultimate conservative difference scheme", Journal of Computational Physics, 135(2), pp. 229-248 (1997).

27. Anderson, W.E. and Wong, N.D. "Experimental investigation of a large scale, two-dimensional, mixedcompression inlet system-performance at design conditions, $\mathrm{M}=3.0$ ", NASA TM X-2016 (1970).

28. Soltani, M.R. and Farahani, M. "Effects of angle of attack on inlet buzz", Journal of Propulsion and Power, 28(4), pp. 747-757 (2011).

29. Soltani, M.R., Farahani, M., and Sepahi Younsi, J. "Performance study of a supersonic inlet in the presence of a heat source", Scientia Iranica, 18(3), pp. 375-382 (2011).

30. Soltani, M., Sepahi Younsi, J., and Daliri, A. "Performance investigation of a supersonic air intake in the presence of the boundary layer suction", Proceedings of the Institution of Mechanical Engineers, Part G: Journal of Aerospace Engineering (2014).

31. Soltani, M.R., Younsi, J.S., Farahani, M., and Masoud, A. "Numerical simulation and parametric study of a 
supersonic intake", Proceedings of the Institution of Mechanical Engineers, Part G: Journal of Aerospace Engineering, 227(3), pp. 467-479 (2012).

\section{Biographies}

Abbas Ebrahimi is an Assistant Professor at the Aerospace Engineering Department of Sharif University of Technology, Tehran. He obtained his Ph.D. degree in Aerodynamics from the Sharif University of Technology, Iran. His research interests include CFD, applied aerodynamics, unsteady aerodynamics, and wind tunnel testing.

Majid Zare Chavoshi earned his BS degree in Aerospace Engineering from Malek-e-Ashtar University of Technology, and MS degree in the same subject from Sharif University of Technology. His main research interests are internal aerodynamics, Compressible Flows, and Computational Fluid Dynamics (CFD). 